\title{
Pulses of Light Accelerate in Free Space
}

\section{A recipe for greatly accelerating a light pulse involves sculpting its spectrum in space and time.}

\section{By Rachel Berkowitz}

ight has a hard speed limit, but this bound can be broken for a light pulse's group velocity-the speed at which the waveform's peak travels. So far, approaches for accelerating pulses, which rely on generating specific pulse shapes, have resulted in only modest speed changes that are a minute fraction of the speed of light. Murat Yessenov and Ayman Abouraddy of the University of Central Florida now show how to rapidly accelerate pulses of light in a vacuum to superluminal speeds or decelerate them to subluminal speeds. The result opens possibilities for novel light-matter interactions and the generation of $x$ rays.

Yessenov and Abouraddy recently developed pulsed laser beams whose spatial and temporal degrees of freedom are inextricably linked and whose group velocity exceeds light speed. Building on those experiments, the researchers sculpted the beam's spectrum in a way that links each component wavelength to the spatial patterning of the pulsed beam. The precise linking determines how the beam's group velocity will vary in space, allowing Yessenov and Abouraddy to control

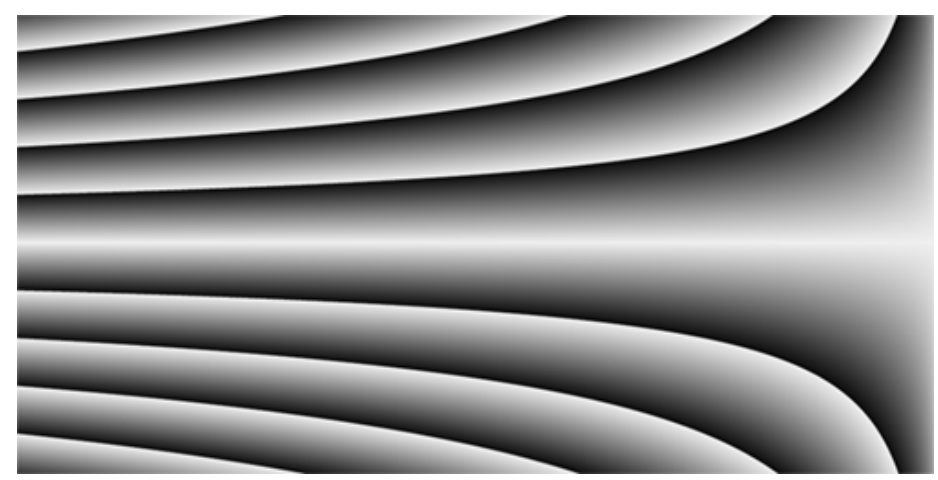

Credit: M. Yessenov and A. Abouraddy [1] when the beam will accelerate or decelerate as it travels.

The researchers observed changes in the pulsed beam's group velocity of up to 1.2 times the speed of light over a distance of only $20 \mathrm{~mm}$-a roughly 10,000-fold boost over previous work. The experimental strategy can be easily extended to create arbitrary acceleration profiles, such as an optical pulse whose velocity first increases and then decreases. Having a way to accelerate light pulses in a versatile configuration could lead to new sources of radiation.

Rachel Berkowitz is a Corresponding Editor for Physics based in Vancouver, Canada.

\section{REFERENCES}

1. M. Yessenov and A. Abouraddy, "Accelerating and decelerating space-time optical wave packets in free space," Phys. Rev. Lett. 125, 233901 (2020). 